\title{
Dialogue sur la poesie de Louis le Caron
}

\author{
SHARON A. ADAMS et CHARLES JOSE
}

\section{Establissement du texte}

Bien que la plupart des critiques qui s'intéressent aux arts poétiques du XVIe siècle ou au genre du dialogue accordent de l'importance à celui de Louis Le Caron, il n'existe aucune édition moderne de ce texte. En fait, il n'existe qu'une seule édition, celle de 1556, dont nous avons consulté trois exemplaires, tous identiques.

1. celui de la Bibliothèque Nationale (R. 18271), lequel nous reproduisons ici;

2. celui du British Museum (8409.e.1);

3. celui de la Houghton Library, Harvard University (FC5.L4944.556d).

LES DIALO- / GUES DE LOYS / LE CARON / Parisien, / A PARIS, / Pour Jean Longis Libraire, tenant sa / boutique en la gallerie, par ou / lon va à la Chancellerie, / 1556. / Avec privilege. In- $8^{\circ} .176 \mathrm{ff}$.

Le dialogue sur la poésie est le quatrième de cinq dialogues dits "du premier livre", dont les trois premiers portent les titres: "Le Courtisan premier, Ou, que le Prince doit philosopher", "Le Courtisan second, Ou de la vraie sagesse, et des louanges de la philosophie" et "Valton, de la tranquilité d'Esprit, Ou du souverain bien". Le dernier s'appelle "Claire, ou de la beauté iiii dialo. desquelz le premier est comme l'argument, ou epitome des autres". La table des matières annonce dix autres dialogues; mais comme il ne semble exister aucun exemplaire de ces dialogues, on pense que Le Caron aurait projeté un ouvrage encyclopédique, en forme de dialogues, qu'il aurait par la suite abandonné. D'ailleurs, dans l'avantpropos au lecteur, l'auteur nous parle des difficultés personnelles qui l'auraient empêché de mener à terme cet ouvrage au moment de sa publication en 1556. A la dernière page l'imprimeur a ajouté: "Parce que la grandeur des dialogues ensuivants de la beauté, et des autres livres merite plus ample volume, on a differé de les imprimer jusques à ce que l'auteur aiant plus grande commodité mette touts ses euvres en lumiere". 
Dans notre transcription du texte, nous avons respecté l'orthographe et la ponctuation de l'édition de 1556 , respectant jusqu'à des orthographes différentes d'un même mot lorsque cela se présentait. Nous avons cependant introduit les modifications suivantes:

1. résolution des abréviations, à l'exception de celles des titres d'ouvrages;

2. différenciation de $i$ et $j$, de $u$ et $v$;

3. substitution de $y$ à $i$ lorsqu'il s'agit du pronom adverbial;

4. généralisation de l'usage de l'apostrophe (quelle remplace qu'elle, l'a remplace la, etc.);

5. introduction de l'accent grave à où, dès, près, après, enaprès; suppression de quelques accents qui constituent des fautes d'impression (celestes au lieu de celestés, fortuites au lieu de fortuités, a au lieu de á); généralisation de l'usage de la cédille au verbe sçavoir;

6. généralisation de l'usage de certains traits d'union là où l'orthographe nous a paru inconséquente (cétui-ci est préféré à cétuici, chosepublique à chosepublique, bienseance à bien-seance, vraisemblable à vrai-semblable, quelquefois à quelque-fois, selon la fréquence la plus élevée); généralisation de l'usage du trait d'union dans l'interrogation;

7. séparation de certains groupes de mots là où l'orthographe nous a paru inconséquente (de quoi est préféré à dequoi, au moins à aumoins).

Toute autre correction est indiquée dans les notes, où nous nous sommes contentés d'identifier les sources antiques (souvent indiquées dans les marges par Le Caron lui-même) et de fournir le sens de quelques mots sortis de l'usage. Tout autre commentaire sur le texte doit paraître dans le prochain numéro de Renaissance et Réforme.

\section{McMaster University}

Ronsard. ou, de la Poësie.

Pensant quelquefois comment la poësie a gaigné en la France le premier honneur, entre toutes les sciences dignes de l'homme noble et vertueux: il m'est resouvenu que par le mesme commencement les autres nations ont parvenu à la gloire [127v] de la souveraine congnoissance de toutes les choses lesquelles l'homme bien né doit embrasser. D'autre part considerant ce que les anciens avoient jugé de l'excellence de la poësie, laquelle plusieurs desirent veoir plus perfaitement representée es 
carmes de noz poëtes: il m'a semblé que ne pourrois mieux exciter les bienheureux esprits de nostre eage, que si je recherchois en la venerable antiquité quelque exemplaire de ma conception, afin de leur donner un meilleur eguillon à plus haultes inventions. Mais l'aiant trouvé j'ai longuement douté s'il pourroit satisfaire aux hommes de plus éveillé et subtil entendement. Toutesfois pour monstrer le chemin aux autres je me suis enhardi d'exposer mon nom au hazard des diverses opinions d'une volage multitude. Socrate disputant avec Alcibiade recite ce carme d'un poëte. ${ }^{1}$ Il avoit bien congneu plusieurs choses: mais mal

$I l$ avoit congneu tout.

Alcibiade n'entendant que vouloit dire Homere ne pensoit que ce vers eust quelque convenance avec leurs propos. Mais Socrate l'interpretant monstre que de sa nature la poësie est pleine d'enigmes, et non vulgaire à châcun. Qui me fait penser qu'elle a esté tant honnorée par les anciens, qu'ilz ne la prophanoient à la multitude: ains $^{2}$ la rendoient admirable par une grave obscurité de sentences. Aussi premierement les Rois, les princes, les prestres et les prophetes estoient seulz [128] qui la traittoient. Encores l'eage commençant à se changer ilz ont longuement retenu et gardé l'honneur aux poëtes: comme Polycrate tyran des Samiens à Anacreon, Heiron Syracusien à Eschile et Simonide, Denistyran de Sicile à Philoxene, Antigone Roi de Macedon à Antagore Rhodien et Arate. Je sçai bien que Hesoide et Homere n'ont suivi les courts des Rois, ou par une injure de fortune, ou par le mespris des vaines pompes et richesses, ou par quelque autre cause. Toutesfois nous lisons en Homere Demodoque avoir esté familier à Alcinoë, et Agamemnon avoir laissé un poëte avec sa femme. ${ }^{3}$ Non moindre a esté la faveur d'aucuns de noz Rois, mesmement de François le vrai Roi de toutes les sciences, envers ceux qui avoient quelque grace et naiveté à bien dresser une rime, combien qu'ilz ${ }^{4}$ n'eussent beaucoup de poësie. Recueillant donc en mon esprit infinis autres semblables discours je ne les ai peu mieux expliquer, qu'en une maniere de familier devis, ${ }^{5}$ lequel desirant embellir et decorer de toutes ses bienseances, à l'exemple des anciens qui avoient accoustumé d'enrichir leurs dialogues de telz ornements, j'ai pensé qu'il representeroit bien sa dignité, s'il estoit discouru par les deux, qui sont au jourd'hui à bon droit reputez les premiers poëtes de nostre tems, Ronsard et Jodelle, avec deux orateurs Pasquier et Fauchet, lesquelz l'excellence de leur esprit, pour la bonne esperance d'eux m'a fait tousjours aimer. Mais si aucun d'eux s'estonne, que je le fai [128v] parler de ce que paraventure il n'a jamais ne dit ne pensé, ou est entierement contraire à son opinion: je croi, que se resouvenant de la coustume des dialogues il ne trouvera estrange, que j'aie emprunté son nom et sa personne. Aussi je les congnoi estre mieux nez, qu'ilz daignassent tirer en la mauvaise part, ce qu'une bienveullance me leur fait franchement 
attribuer. Partant j'ai fait ainsi commencer Ronsard, comme si tout le propos eust esté disputé en la grande salle du palais roial de Paris, la plus renommée et excellente ville du roiaume: En laquelle souvent les compagnies des hommes doctes s'assemblent et se pourmenants devisent quelquefois de choses graves et serieuses. RONSARD. Je m'esbahi (messieurs) comment le bruit de tant de voix tumultuantes ne trouble le repos de voz tendres et delicates oreilles, lesquelles aucunefois amignar$\mathrm{dez}^{6}$ des amoureuses douceurs de la poësie. JODELLE. Nous estions en mesme propos traittans la raison, que le seigneur Pasquier en avoit.donnée, laquelle il te recitera mieux. PASQUIER. Je disois (seigneur de Ronsard) que l'accoustumance conjointe avec un gracieux espoir du gain nous avoit $\mathrm{si}^{7}$ bien faits et stillez ${ }^{8}$ au tumulte, qu'il ne nous estoit plus qu'un plaisir: à l'exemple du bruit des disputes scholastiques, ausquelles celui qui est façonné ne les trouve enaprès ${ }^{9}$ aucunement fâcheuses. Mais laisson ce propos, au lieu duquel je te prie nous donner [129] un meilleur goust des delices de la poësie: car je pense que de ta riche veine, comme de la source d'Helicon sont écoullez en la France infins ruisseaux, desquelz les jeunes poëtes s'éforcent d'épuiser les plus beaux carmes de leurs braves rimes: et naissent d'une de tes inventions aussi drus et frequents, que d'une des dents du Dragon tué par Jason armé des arts de Medée, il naissoit (comme aux fables) de petits hommeaux. RONSARD. Je ne suis tant amoureux de moi-mesme, que je face céte louange mienne: car elle seroit mieux reservée au seigneur Jodelle, duquel esperons choses admirables. Mais pour ne perdre le tems en telles caresses coustumieres entre les amis, je me veux éforcer de te satisfaire, si ce propos ne desplaist à la compagnie. JODELLE. Je ne pense point (Ronsard) qu'aucun de nous se puisse ennuier de t'ouir parler principalement des choses si grandes et convenables à nostre commune estude. RONSARD. De touts les genres des hommes, qui ont jadis fait reluire à l'utilité publique quelque profession, quelque art ou quelque science digne d'immortelle memoire, nous lisons les poëtes avoir esté les premiers: de sorte que de touts les secrets des choses plus cachées en nature, qui sont parvenus aux hommes du moien eage et de cétui-ci, il n'en faut donner la gloire à autre, qu'à la poësie. Non seulement les fables (lesquelles toutesfois sont les imitations de la verité) ains aussi les histoires font foi suffisante que le siecle heureux estoit doré et enrichi des [129v] poëtes, qui chantoient la grandeur de Dieu, les vertus des Heroës, et excitoient châcun à faire les chose honnorables à l'immortalité de leurs noms. Ainsi leurs chants estoient une vraie doctrine de bien-vivre, ou plustost une sainte fureur qui inspiroit les hommes à congnoistre le lieu de leur celeste origine, et dresser à ce but toutes leurs pensées. A céte cause ilz sont appellez mesmement par Platon les peres et Capitaines de sagesse, et les premiers laboureurs des vertus. ${ }^{10}$ Le commencement 
de la societé politique, laquelle au jourd'hui châcun cherit, est l'une des marques de l'excellence de la poësie. Car Amphion et Orphée par le son de leurs lires ont assemblé les hommes brutaux et sauvages, qui n'estoient beaucoup differents des arbres et des pierres. Qu'est-ce qui eust peu mieux attirer à une compagnable civilité les hommes épars et impolis, que la plaisante et harmonieuse douceur de la lire? Il failloit reveiller ces premiers esprits endormis en une horreur de vie par l'attraiante volupté de ce qui est plus agreable aux oreilles. Qui est l'homme si mal né et de si grossier et lourd entendement, qui ne sente en soi toutes ses parties plus nobles se ravir au chant d'une gracieuse chanson, et y prendre quelquefois si grand plaisir, qu'il semble estre transporté de tout autre pensement? Je ne dirai quelles lois estoient anciennement escrites en carmes et les oracles rendus en vers: mais je ne puis passer ce que dit nostre Pindare les mortelz reluire par les poëtes, et l'hinne estre semblable à l'or, lequel quand [130] on fait fondre, monstre touts ses raions resplendissants: ${ }^{11}$ ainsi l'hinne celebrant les faits des hommes vertueux les rend illustrement semblables aux Heroës et aux Rois. Puis-que nostre vie destinée à la mort est bornée d'un si brief eage: quel autre espoir nous peut mieux consoler, que de chercher les moiens pour perpetuer nostre nom: afin que la posterité congnoisse que nous aions esté quelquefois? Combien que pour plusieurs autres causes l'ame semble estre divine et immortelle: toutesfois pour céte raison principalement les hommes en ont eu plus certaine opinion, qu'on veoit les esprits des excellents personnages presentir tellement la posterité, qu'ilz ne semblent rien penser ne regarder, que les choses eternelles. Céte congnoissance nous a esté revellée par les poëtes, mesmement par Pindare, lequel a chanté que les hommes sont semblables aux Dieux quant à l'Entendement, et ceux qui sont privez de céte vie (combien que leurs corps soient couverts de poudre) jouissent d'une bonne partie des faits vertueux de leurs enfants et neveux: mais la vertu seulement, qui est congneue par les riches vers, dure à perpetuité. ${ }^{12}$ Tu veois la grandeur de la poësie, laquelle peut exprimer tout ce qu'elle veut et en telle perfection, que les autres arts l'admirent plustost, qu'ilz ne l'imitent. Qu'on face les Legislateurs, les sages et philosophes tant grands qu'on voudra, s'ilz confessent devoir aux poëtes tout ce qu'ilz ont de meilleur, ilz les doivent recongnoistre comme les plus honnorables: s'ilz nient, tres-facile sera de les convincre de lar-[130v]cin. Quelz autres plus anciens philosophes peuvent reciter les Grecs vantarts, que Line, Musée, Anaxagore, Empedocle, qui touts ont escrit en poësie? A mesme fin elle tend que la philosophie, à sçavoir d'exercer et orner l'esprit de l'homme. Elle peut aussi bien s'attribuer la science des choses divines et humaines, que l'autre se vante de l'embrasser: Et n'est grandement diverse de la peinture de Polygnote ou Zeusis non moins doctes es autres arts, qu'excellents en 
leur profession: Lesquelz se proposoient deux choses, l'une de leur art, et l'autre qui dependoit de la vertu et congnoissance des autres choses. C'estoit bien de leur art de representer au vif l'image du corps, qu'ilz dessignoient de peindre: mais il venoit de leur vertu et plus haulte science d'exprimer la perfection d'une beauté en toutes ses graces et bienseances. En telle splendeur faut veoir la poësie: car outre le sujet qu'elle se propose à imiter, elle doit comprendre tout ce qui est d'exquis, de rare et de perfait es sciences non indignes de sa gravité. Je veux recueillir deux ou trois lieux des odes de Pindare pour les comparer aux sentences de Platon, que tant on appelle divin. Ce grand philosophe est honnoré de châcun pour avoir en plusieurs de ses dialogues disputé, que la sagesse et la vertu ne viennent de nature, et ne sont aquises par art, ains données par l'heur de la puissance et volunté divine. ${ }^{13} \mathrm{Ce}$ que Pindare avoit devant lui chanté en la 9. ode des Olympies. [131]

Mais les bons et les sages

Du grand Dieu seulement.

Strophe 2.

\section{Proviennent.}

En la mesme ode.

Plusieurs par leurs vertus

Aquises de science

Se sont fort debattus

De gaigner l'excellence

D'un renom honnorable:

Mais ce qui est sans Dieu

Par destin miserable

Est obscur en tout lieu.

En l'ode 3. des Isthmies parlant à Jupiter.

$L$ 'illustre vertu par ta grace

Compaigne aux mortelz les embrasse.

En l'ode 5. du mesme livre.

La force des mortelz est de Dieu. ${ }^{14}$

Ce que dit Platon de Dieu, qu'il est la perfaite cause et l'auteur de toutes choses, qui gouverne le monde, prospere et enrichit les hommes, le commencement, le milieu et la fin de tout: ${ }^{15}$ a esté premierement escrit par Pindare disant que Jupiter celà et celà gouverne, seigneur de toutes choses: en lui est la fin et entier accomplissement des euvres que le commencement des choses humaines croist tousjours par l'inspiration de Dieu, lequel selon sa volunté perfait tout, eslevant quelquefois les choses petites et abaissant les haultes: et rend les choses faciles, lesquelles [131v] estoient contre toute esperance. ${ }^{16}$ La doctrine de Platon est qu'es choses humaines, perissables et sujettes à divers changements ne se 
trouve rien de certain: ${ }^{17}$ Pindare dit qu'à l'entour de la pensée humaine pendent infinis erreurs, et la felicité ne dure longuement: ains l'esperance tourne plusieurs choses desus, et derechef plusieurs desous ne trouvant aucune asseurance en l'evenement de son entreprise. ${ }^{18}$ Platon fait souvent disputer Socrate que le seul sage est riche. ${ }^{19}$ Pindare.

Excellente est la richesse, Laquelle est jointe à la sagesse.

En la 8. du mesme livre.

Quand quelcun s'enrichit sans peine,

Il semble aux ignorants avoir.

Epode.

Par l'art de la prudence humaine

Aquis si grands biens et pouvoir.

Mais des hommes la force est vaine:

Ains Dieu, qui or' hausse en grandeur,

Or' baisse par raison certaine,

Est celui, duquel vient tel heur. ${ }^{20}$

Châcun d'eux parle du destin de la mort en mesme sorte, disant ne le riche ne le pauvre, ne le vivant sans gloire ne l'ennobli de renommée pouvoir fuir le tombeau de la mort. ${ }^{21}$ Pindare en l'ode 2. des Olimp.

Les mortelz n'ont l'heure assignée

De la mort, et avec un bien

Perdurable n'est ordonnée [132]

La fin du jour, qu'on attend sien. ${ }^{22}$

Platon au dialogue du roiaume et au 8. de la Chose-publique traitte que l'estat populaire, ou gouvernement de la multitude, combien qu'il semble avoir quelque apparence de perfaite administration, ne peut toutesfois estre une vraie Chose-publique. ${ }^{23}$ Laquelle opinion convient à ce que dit Pindare en l'ode 8. des Olympies.

Car où est une multitude

Diverse en avis et estude,

Et où se retirent sans fin

Variables choses, en vain

On penseroit estre facile

De discerner un droit utile:

Il fauldroit quelque loi des Dieux, Qui vivent immortelz aux cieux. ${ }^{24}$

Mais qu'est-il besoing de plus long propos? J'ai le tesmoignage de Platon mesme, lequel en un lieu recite que Pindare a dit la loi estre la Roine de touts les mortelz et immortelz et icelle conduire le droit d'une main tres-puissante: ${ }^{25}$ et au premier dialogue de la Chose-pub. il escrit qu'à celui qui n'est à soi-mesme coupable d'aucune injure, se presente tousjours la joieuse esperance, la tres-bonne nourrice de la vieillesse, 
comme dit Pindare. ${ }^{26}$ Mais non seulement de cétui-ci, ains aussi de Theognide, Homere et infinis autres divins poëtes Platon a retiré le meilleur de sa philosophie. Nous lisons qu'il s'est grandement aidé des labeurs d'Epicharme Comique, mesmement [132v] es disputes du bien, des Idées et de la difference des choses qui ne sont comprises que par l'Entendement et celles qui tombent au sens. ${ }^{27} \mathrm{Je}$ parlerois et de lui et des autres philosophes plus amplement, si je ne doutois que par trop grande admiration des anciens je feisse injure à ceux de nostre eage, lesquelz j'accuserois d'ignorance, si me defiant de leurs forces je donnois à iceux tout l'honneur des sciences. Car j'espere quelquefois de veoir nostre France si riche, que les vantances ${ }^{28}$ des estrangers n'auront plus de lieu envers elle. Les Poëtes donc (pour retourner à mon premier propos) n'ont rien oublié en leurs vers de ce qui pouvoit estre congneu en céte grandeur de l'universelle nature. Mais comme toutes les choses desirent de retenir leur perfection, et s'avilissent si quelquefois est diminué de leur excellence: ainsi les poëtes (lesquelz le peuple estimoit prophetes des Dieux) pensants estre indigne de prostituer leurs sacrées inventions au prophane vulgaire, les ont voulu couvrir de fables: afin qu'elles ne feussent entendues, que des plus sages et doctes. Toutesfois si naïvement elles imitent et representent la nature, qu'elles semblent estre tirées d'elle: les autres sont plus graves et élongnées de l'opinion commune, sous lesquelles aussi sont cachées les plus haultes et excellentes choses, mais si obscures, que l'ignorante multitude eust mal fait son proufit, si autrement elles eussent esté revelées. En quoi les poëtes me semblent avoir pru- [133] demment regardé à la nature de l'esprit humain. Car rien n'est qui plus l'exerce en ses conceptions, qui lui apporte plus grande volupté et qui le rende plus content, que de discourir les choses plus separées de la congnoissance du vulgaire. Quel grand plaisir se donne l'esprit, quand ravi et abstrait des pensements terrestres il cherche et recherche franchement, invente, conçoit, entend, traitte et dessigne infinis discours, pour trouver la verité, de laquelle la subtile fable lui donne quelque amorce? L'épreuve journaliere fait foi de ce que je di. L'esprit est si noble et haultain, qu'il se monstre plus chiche épargneur d'honnorable louange envers les choses que present il regarde, que celles qui de lui sont plus élongnées. J'ai leu quelquefois en un poëte, que tant soit la beauté belle, si elle n'est parée de riches ornements, de nul sera congneue et estimée: aussi tant soit la sentence excellente, si elle n'est enrichie d'inventions bien agensées et appropriées au sujet qui lui donnent grace, couleur et autorité, ne faut esperer qu'elle soit recueillie de telle faveur, que paravanture elle merite. J'admire non sans cause les poëtes, lesquelz se proposoient de contenter et les plus excellents et le commun. Car si les secrets cachez et enveloppez de leurs rares inventions ne pouvoient estre de châcun entendus: au moins le plaisir du 
discours chatouilloit et les uns et les autres, se rendant digne d'estre embrassé de touts. [133v] Comme le vulgaire ne peut facilement porter la grandeur des plus puissants, s'ilz ne remettent beaucoup de leur gravité: ne le riche plaire aux pauvres, s'il ne s'abaisse jusques à s'accommoder aucunement à eux, ne la vertu estre bien receue de la volupté, si elle n'est couverte de quelque fard: Ainsi le philosophe quelque utilité qu'il puisse apporter à la multitude, pour sa fâcheuse severité est incontinent repoussé: Mais le poëte duquel on n'a telle opinion, est de châcun aimé, prisé et honnoré, non toutesfois qu'il soit sans proufit. Nous voions les medecins quand ilz veulent presenter au malade fâcheus et difficile bruvage de saveur amere, avoir de coustume de le deguiser par quelque douce liqueur. En mesme maniere l'ancienne philosophie a gaigné son premier honneur par le voile et couverture des fables, des carmes et des chants, desquelz elle fardoit la gravité de ses sentences. Je rejette avec Pindare les fables bigarrées de variables mensonges, plains d'inutiles et vaines moqueries: ${ }^{29}$ celles me plaisent seulement, qui ont une exquise imitation de la nature des choses, et avec la delectation l'utilité conjointe. Aucuns escrivent la fable estre en la poësie, ce que la couleur en la peinture, laquelle a plus de force que la ligne, pour faire regarder l'image bien tirée: aussi en l'euvre poëtique cette vraisemblance ornée de fables rend aucunement estonnez ceux qui l'oiënt, ou [134] lisent: et avec meilleure grace, qu'une trop recherchée elegance de sentences et de vers. Phidias se proposant de faire l'image de Jupiter ou de Minerve n'addressoit son projet à aucun, pour en tirer de lui la semblance: ains une Idée de la souveraine beauté estoit engravée en son esprit, à l'imitation de laquelle il dressoit l'art et la main. Le poëte se doit encores moins asservir à quelque sujet, pour entierement le reciter tel qu'il a esté fait: ains comme rapportant ses conceptions à l'université des choses discourir ce qui a peu estre fait, ou a esté vraisemblable, ou grandement necessaire, et le descrire de telle perfection, que rien ne soit en lui qui n'ait sa bienseance tant admirable, que la verité semble plustost l'avoüer sien que le fait mesme. Qui voudroit estimer le poëte à cause des vers qu'il escrit, ne donneroit beaucoup de gloire à la poësie: car ne se trouveroit si petit rimeur, qui ne se nombrât incontinent entre les divins poëtes: ce qui seroit trop absurde et messeant. Comment est-ce qu'une science tant sainte, tant divine, tant pleine de souverains et sacrez misteres se laisseroit familierement traitter par ceux, qui ne s'eleveroient plus hault, qu'à servir à l'applaudissement du vulgaire? En quoi est-ce que la grandeur d'un gentil esprit pourroit plus noblement reluire, qu'à feindre, concevoir et representer les moeurs, les afections et les dignitez des personnes: et d'infinis sujets et arguments, qui se presentent, choisir [134v] un qui mieux convienne et semble estre né avec la mesme chose? Mais non seulement les poëtes combien que les premiers: ains aussi les philosophes, 
orateurs, histoiriens et auteurs dignes de nom ont enrichi leurs inventions de diverses sortes de fables. Qu'est-ce qui rend tant admirable la faconde de Platon, si non la majesté des sentences decorées de si haults secrets? La fable d'Hercule tant souvent repetée et mesmement par Socrate, monstre quelle fin se doit proposer l'homme, et par quelz moiens y aspirer. ${ }^{30} \mathrm{Je}$ ne puis me persuader qu'il eust esté possible au plus subtil philosophe, au plus eloquent orateur, au mieux discourant historien de disputer, dire, ou reciter en telle grace les afections, pensées, deliberations, entreprises et difficultez qui se presentent à l'homme non encores asseuré d'une prudence virile. Que dirai-je de la fable de Promethée? Le feu celeste, lequel il ravit du ciel pour l'inspirer aux mortelz, n'est autre chose (comme aussi Platon escrit les anciens avoir declairé es oracles) que l'Idée, et la pure, subtile et divine congnoissance des secrets, que Dieu a voulu estre cachez au vulgaire. ${ }^{31}$ Je pourrois aussi parler du muable Prothée, qui se changeoit en diverses formes, lequel Orphée dit en ses Hinnes avoir premier enseigné les commencements de toute nature: ${ }^{32}$ tellement que plusieurs ont entendu sous son nom la premiere matiere, laquelle et les poëtes et les philosophes dient avoir [135] esté au commencement confuse, sans ordre, sans forme et sans disposition: et depuis separée et ordonnée en diverses especes avoir constitué ce monde tel qu'il est. Aussi Homere appelle la fille de Prothée, Eidothée, comme celle $\ddot{\epsilon} \iota \delta o \varsigma ~ ๕ ̌ \mu \alpha \sigma \tau o \nu \vartheta \epsilon ́ \alpha \nu$, laquelle avoit presenté l'objet de châcune forme ou Idée. ${ }^{33} \mathrm{Si}$ je voulois reciter ce qu'on dit d'Atlas, lequel feignent les poëtes soûtenir le ciel de ses espaulles: et discourir la fable de ses filles, lesquelles enseignées en la congnoissance de nature ont recueilli des precieux pommiers des jardins, qu'elles labouroient, les pommes d'or, c'est à dire, la science non moins resplendissante et riche, que l'or: j'aurois asses ample sujet, pour vous detenir le reste de céte matinée. Mais laisson l'antiquité en sa gloire, car nostre France plus heureuse laquelle jadis s'espovantoit des fieres et vanteresses imaginations d'elle, consacre maintenant à la memoire les plus haultes et excellentes inventions, desquelles la poësie devoit estre perfaite. La felicité d'esprit que châcun sçait estre le premier heur du poëte, reluit plus illustrement en la grace et naïveté Françoise, qu'en l'orgueil enflé des Grecs, des Romains, ou des Italiens. Toutesfois souvent la France se peut plaindre d'enfanter je nesçai quelz monstres, qui semblent estre plus nez aux estrangers, qu'à la patrie. Puis que de la sage et industrieuse nature, ou de l'art naturel vient la noble poësie: allieurs ne faut chercher où plus liberale- $[135 \mathrm{v}]$ ment elle se presente, qu'en céte grandeur et liberté françoise, prodigue d'admirables et riches inventions. JODELLE. J'ai tousjours ainsi pensé de la France, la reputant aucunement ingrate envers les bons esprits, ses vrais enfants, lesquelz peu, ou plustost rien au regard des estrangers elle n'honnore. Mais j'ai accoustumé de parler de la 
poësie autrement que des autres arts: par ce qu'elle a je nesçai quoi de divin, qui surpasse les vulgaires conceptions. Comment peut estre l'esprit tant bien né, tant diligemment institué, qu'il invente les choses plus grandes que la nature humaine et la prudence de l'art? Je croi que c'est une celeste fureur, laquelle nous inspire et ravit sus les communes pensées, et dedie noz purs esprits au temple des Muses, c'est à dire, les fait rechercher (comme interprete Platon) ${ }^{34}$ les secrets des choses épandues par nature. Si les anciens n'eussent esté agitez de céte inspiration, le nom de fureur n'eust jamais eu quelque autorité: mais sentans en eux que leurs conceptions estoient vaines sans la chaleur qui renforçoit leur naturel, ilz ont congneu céte fureur venir de Dieu, et estre plus excellente, que la prudence humaine. L'homme forcené par la violance d'une ardante maladie semble estre transporté hors de la raison, et pres-que semblable aux bestes: au contraire pourquoi celui, lequel la haultesse des imaginations ravit et abstrait des estudes, dessins et [136] exercices du commun des hommes, ne sera estimé plus excellent que les autres, comme tenant daventage du celeste? Qu'est-ce autre chose céte fureur, si non l'inspiration des Muses, laquelle souffle en l'ame pure et non souillée des vaines afections, une sainte chaleur qui l'embraze toute de divinité? Ce que les vrais poëtes revelent par leurs chants, quand la fureur commençant à les ravir les presse de si terrible force qu'ilz tremblent de fraieur, tresaillants de l'horreur, qui les estraint, leurs cheveux dressez, et leur esprit plus hault elevé, que la congnoissance du vulgaire. Qui dira donc céte aflation ne venir d'une secrette vertu descendante du ciel? Mais qui voudroit sans la fureur des Muses approcher (comme dit Platon) aux portes poëtiques sous céte esperance que par l'art il pourroit estre bon poëte: ${ }^{35}$ icelui certainement seroit imperfait, et sa poësie au regard de celle qui est pleine de fureur, s'évanouirroit. Si pour l'amour des choses divines et humaines le philosophe s'enorgueillit d'immortelle renommée: pour l'imitation d'elles le poëte me semble plus digne de l'immortalité mesme. Bien me plaist la sentence de Socrate, lequel disputant avec Iön Rhapsode et recitateur des vers d'Homere prouve l'art ne pouvoir faire que les poëtes chantent les carmes riches et ornez d'excellentes et haultes inventions: mais seulement l'inspiration de la divine fureur, laquelle il [136v] compare à l'Emant: ${ }^{36}$ ce qui a esté imité en ces vers par un de noz amis.

Comme l'Emant non seulement attire

Le fer touché: mais l'autre et l'autre aussi

Tiré attire en longue suite ainsi

Qu'ensemble à touts la mesme force inspire:

Ainsi espris le poëte tres-saint

De la fureur divinement infuse, ?ar la vertu d'une celeste Muse, 
Vient saisir l'un, qui l'autre aussi attaint.

Qui veit jamais des anneaux l'entresuite

Touts hault-pendus, et ensemble enchenez:

Celui peut veoir des hommes estonnez,

Ravis d'esprit l'inspirante conduite.

Mais je ne trouve grande raison en ce que Socrate dit la fureur ravir plustost un inepte et mauvais poëte, qu'un prudent et de noble esprit: afin qu'il ne semble que l'euvre d'icelui vienne de l'art humain, ains de l'infusion et vertu celeste. ${ }^{37}$ Car je pense les esprits d'autant plus qu'ilz sont honnorez des dons et de nature et de l'art, approcher plus près à la divinité. J'ause encores dire daventage, que sans l'inspiration et instinct de Dieu telz dons sont froids, sans grace, sans force et sans valeur. Comment les bons poëtes peuvent estre autrement distinguez des mauvais, si non par céte raison, que les uns saisis de la sainte fureur des Muses chantent les vers surpassants les humaines conceptions, et embellis de l'admirable gravité de toutes les sciences: les autres [137] par art, par sueur et par peine s'éforcent de faire, ce que le commun esprit des hommes peut comprendre, travaillants en vain d'aspirer à l'accomplissement de quelque euvre memorable. Je ne doute qu'on m'objettera que le poëte remis et delaissé de la fureur qui l'agitoit, ne peut à grand'peine recongnoistre les graves et obscures inventions, desquelles la chaleur celeste le remplissoit: comme si céte inspiration n'estoit qu'un vent, lequel soufflé ne laisse de lui aucune trace. De quoi on pense avoir certain argument des carmes de noz plus superbes et audacieux Poëtes, lesquelz sont quelquefois au commencement enflez de telle haultesse, qu'ilz semblent vouloir surpasser la grandeur du Soleil. Mais peu à peu ilz s'abaissent au plus humble et abjet vulgaire sans rien retenir de leur premiere fureur. Toutesfois je ne sçai sus quelle vraisemblance céte opinion peut estre appuiée: car de raison elle n'en a point. Aussi faudroit dire la nature ou l'art, estre de plus grande felicité, que l'instinct venant de Dieu, ce qui n'est religieux de penser. L'heur de nature apporte tel plaisir, qu'il excite tousjours à esperer un bien plus honnorable: l'art donne merveilleux contentement à l'esprit, et console ses labeurs de favorable attente. Mais que seroit-ce, si avec la relâche de la fureur divine laquelle inspiroit toutes les sciences, incontinent l'ardeur du ciel estoit esteinte? Ce n'est ainsi qu'on doit juger de la puissance des Muses: de- [137v] puis qu'unefois ravissent l'esprit à elles, tousjours l'entretiennent en une grandeur digne de leur divinité. Ainsi elles ont fait exceller Orphée, Amphion, Homere, Pindare, Virgile, Horace et autres: mesmement en nostre eage plus fleurissant en bons poëtes, que nuls des tems passez. Quant à ceux, lesquelz se feignent par moments estre époints ${ }^{38}$ d'une celeste et violante agitation, et incontinent à eux revenus se mescongnoissent, ilz me semblent miserablement tourmentez de quelque maladie, qui les rend ainsi 
par intervalles furieux, sots ou insensez. Mais la fureur des Muses n'inspire rien, qui ne soit tout perfait, divin et rare. A céte cause Pindare parlant (à mon avis) des poëtes chante ainsi.

Des Dieux les enfants immuables Prosperent tousjours indontables. ${ }^{39}$

Partant le poëte inspiré des Muses, et haussant le vol de son meilleur esprit a congneu l'ordre, la convenance et l'harmonie de l'Univers, les mouvements, les accords et les dispositions non seulement des huit cieux ou globes, ains aussi des intelligences plus haultes et surnaturelles. Daventage céte sainte fureur l'a tant fait divin, qu'il a peu comprendre en une certaine raison de nombres et de mesures les proportions et consonances, aussi bien de tout l'Univers, que de ses parties entre elles. A quoi jamais le sens commun des mortelz n'eust peu et ne peut aucunement attaindre. Ainsi separant ses conceptions de l'opinion du sens variable, douteux et incertain, il a dressé le chemin aux grands esprits [138] pour parvenir à la vraie science. Qu'est-ce qui pourroit apporter plus seure et ferme congnoissance des choses divines et humaines, que la recherche de l'harmonie, qui entretient le monde: et fait que rien ne soit fortuit au ciel, ne rien en la terre, ne rien aussi es autres elements, sans cause et sans quelque raison de nombre et mesure? ne faut penser que par autre maniere les hommes aient peu entendre la vertu de temperer et accorder les afections de l'ame, que par l'imitation de l'ordre et bienseance de l'harmonie celeste, laquelle premierement a esté par le poëte exprimée es nombrées, mesures, rimes et proportions de ses vers: tellement qu'on le peut dire le premier auteur de la Musique, non de céte vile et corrompue, laquelle sans raison degoise un amas de chansons: mais de celle, laquelle par la science des nombres rend l'harmonie bien proportionnée de toutes ses mesures et consonances, Les anciens philosophes et entre autres Pythagore ont escrit toutes les choses consister en nombre, duquel venoit l'harmonie: ce qu'ilz n'avoient appris du son des marteaux (quoi que plusieurs l'aient ainsi recité) ains des observations qu'ilz faisoient tant de l'accord des choses celestes, que de la convenance des actions humaines temperées par un consentement des desirs corporelz obeïssants à la raison. Mais qui a esté le moulle, l'objet, ou le patron de si admirable et exquise recherche, si [138v] non la poësie? Châcun sçait la musique estre appellée des Muses, sous les noms desquelles sont comprises toutes les sciences: comme il est vulgaire mesmement entre les Grammariens. Partant les poëtes (qui seulz se renomment des Muses, qui sont d'elles plus que nulz autres cheris, et rien ne pensent ne conçoivent que par l'instinct et aflation d'elles) ne peuvent estre autres, que souverains en la congnoissance de toutes les choses. Je n'ignore que plusieurs abusent des noms et des Muses et de la Musique. Mais les autoritez de Pythagore, Timée Locrien et Platon sont telles envers moi, que les autres ne peuvent 
rien. Ilz sont touts de céte opinion que la musique est le plus excellent exercice de l'esprit pour accorder, composer et moderer les moeurs de l'ame en une gracieuse convenance, à l'imitation de l'ame de l'Univers. ${ }^{40}$ Telle est aussi la poësie non controuvée à quelque vaine et deraisonnable volupté, ains donnée des Muses pour la consolation de l'esprit. Timée escrit. Puis-que la Musique et la philosophie Duchesse et gouvernante d'elle sont constituées et des Dieux et des lois, pour corriger les esprits: elles deux ensemble excitent, suadent, ${ }^{41}$ et aussi contraignent la partie de l'ame sauvage et cruelle à obeir à la raison: en maniere que l'ire soit facile et bien-traittable, et la cupidité tant moderée et tranquile, que sans la raison elle ne s'emeuve. ${ }^{42}$ Ainsi donc il conjoint la Musique avec la philosophie. Si plus diligemment [139] nous voulons regarder, la Musique est la vraie philosophie, et la philosophie la vraie Musique: mais l'une et l'autre ont leur commencement de Poësie, ou plustost sont la mesme Poësie. J'ai appris des plus celebres auteurs que l'ancienne poësie tant la Scenique que la Lyrique estoit toute composée de choeurs, ou troupes de personnes chantantes, comme depuis a tousjours esté la Tragedie. Et certainement si les carmes ne sont façonnez à la raison harmonique, ilz n'ont nulle grace et nulle bienseance. Qui est la cause, qui m'a plus facilement persuadé, que si la Musique n'a esté recueillie des nombres poëtiques, au moins elle a esté inventée pour eux. Je sçai bien qu'au lieu de la vraie et excellente Musique, telle que jadis les sages embrassoient, a tousjours depuis couru comme un vent de ville, quelque vulgaire et impudente chanterie ${ }^{43}$ (j'use de ce mot, que le commun a receu à mesme propos). Athenée recite qu'Aristoxene escrit qu'anciennement estoit une marque d'ignorance de meriter l'applaudissement du vulgaire: mais après que la Musique a esté toute destinée aux theatres pour complaire à une sotte multitude, elle est devenuë tant vile, que les nobles esprits la dedaignants l'ont rejetté aux mercenaires farceurs, menestriers, et je nesçai quelz autres encores plus abjets. ${ }^{44}$ Toutesfois la sagesse des Grecs a esté principalement traittée par les principes et theoremes de Musique, suivant l'o-[139v] pinion de Pythagore, qui avoit par telles raisons demonstré la composition de l'Univers. Platon appelle celui le vrai Musicien, lequel selon la melodie Dorique (laquelle est Grecque et resonne de plus excellente et magnifique harmonie) tempere sa vie d'une concorde de parolles et de faits, ${ }^{45}$ disputant de la sagesse et de la vertu, sans offenser les oreilles des hommes bien nez. Les anciens constituoient trois $^{46}$ Muses Melete, Mnime et Aöide, trois tons Hy pate, Mese et Nete, trois parties de l'ame, la cupidité, l'ire et la raison: ce qu'ilz avoient appris d'un certain secret, lequel es cadences, reprises et rimes des vers les poëtes avoient accoustumé de donner au nombre de trois pour quelque cachée perfection, qui est en lui, comme encores nous voions es Odes, Hinnes et autres chants lyriques, lesquelz me semblent retenir 
daventage de l'antiquité: parce qu'ilz estoient chantez en la gloire des Dieux Heroës, Rois et Princes. Et pour céte cause les poëtes distinguoient ainsi leurs chants, afin qu'ilz feussent marques immortelles de l'observation qu'ilz avoient faite des deux mouvements du ciel: l'un qui est de tout le monde d'orient en occident, et l'autre contraire à icelui d'occident en orient, propre aux spheres et globes particuliers: et aussi de l'immouvable fermeté de la terre. Depuis le nombre des Muses a esté augmenté jusques à neuf, à cau-[140] se des neuf harmonies, lesquelles accordent l'ordre des huit cieux, et le font consoner avec celui de l'Univers, suivant la sentence des Pythagoréans, qui constituoient une ame universelle de toute céte machine, laquelle ilz nommoient le premier nombre ou le commencement des nombres, et la souveraine harmonie, ou la perfection des harmonies: ainsi que j'ai peu recueillir des commentaires, qui nous restent aujourd'hui de leurs doctrines, mesmement des Platoniciens qui les ont principalement imitez. ${ }^{47}$ Partant l'harmonie de l'ame de l'Univers est representée par Calliope, laquelle nous pourrons dire estre tirée de $\kappa a \lambda \sigma \nu$, que Socrate interprete beau, et prouve estre le vrai surnom de la prudence. ${ }^{48}$ Les autres Muses sont ainsi ordonnées aux huit spheres: Uranie au huitiéme globe orné d'une infinie multitude d'estoilles resplendissantes, lesquelles semblent estre fichées au ciel, tant sont constantes et arrestées en leurs cours, mouvements et distances. Polymnie accompagne Saturne, Eraton Jupiter, Terpsichore Mars, Melpomene le Soleil, Thalie Venus, Euterpe Mercure et Clion la Lune: lesquelles les poëtes anciens ont feint estre filles de Jupiter et Mnemosine (c'est à dire Memoire) engendrées par neuf baisers. Mais le baiser de Jupiter signifie aux anciens l'aflation divine, laquelle infuse en la memoire de [140v] l'esprit humain inspire en lui toutes les sciences. Je ne veux philosopher sus les fables du cheval Pegase, de la fontaine Cabaline, ne du mont Parnasse si souvent repetées: Toutesfois ne faut oublier qu'à l'imitation des harmonies des sept planettes divisées en sept spheres, les Musiciens ont diversifié les tons jusques au nombre de sept, et ont prins le huitiéme qu'ilz appellent double ou octave, sus la consonance du huitiéme ciel. Mais ils n'ont encores attaint à la resonnance de Calliope l'ame de l'Univers, à quoi toutesfois ilz doivent aspirer pour la perfection du nombre de neuf. PASQUIER. Voz discours merveilleusement me contentent, non seulement pour le plaisir du devis, ains aussi plustost pour l'utilité de la congnoissance qu'ilz apportent. Mais repensant plus diligemment à iceux je nesçai quelle discorde s'émeut en mon esprit pour chercher la raison, qui a persuadé à ce grand philosophe Platon de bannir de sa Republique touts les poëtes, et mesmement le plus excellent d'eux Homere, lequel toutesfois en plusieurs lieux il honnore, l'appellant le divin poëte, ${ }^{49}$ et excite châcun à le suivre et embrasser, comme le vrai Capitaine des sages. Si je voulois reciter toutes les sentences d'Homere, 
lesquelles Platon confesse avoir tirées de lui pour en decorer sa philosophie: je croi qu'à grand'peine un an me suffiroit. Socrate condamné à mort, devant que de boire le [141] venin eût un propos avec ses disciples de l'immortalité de l'ame, auquel il usurpe ces carmes d'Homere.

Lors frappant sa poitrine Ulisse ainsi parla, Endure encor' mon coeur par ta force celà.

Car tu as autrefois porté plus dures peines. ${ }^{50}$

Pour monstrer que la nature de l'ame n'est semblable à celle du corps, ne tant liée aux afections d'icelui, qu'elle se doive flechir et assujettir à elles. Quant à l'opinion des philosophes, de laquelle tu disputois maintenant (seigneur Jodelle) il me semble que l'harmonie des sons celestes accordez d'un perpetuel mouvement a esté mieux exprimée par Homere, que par nul autre. Il chante en céte sorte d'Apollon (qui est aussi appellé le Soleil).

Pendant qu'il se mouvoit, ses fléches resonnantes

D'un grand son s'élançoient de courroux estonnantes. ${ }^{51}$

Car les anciens pensoient que les corps celestes et principalement le Soleil en céte vehemente course qu'ilz font sans repos et entre-cesse ${ }^{52}$ d'orient en occident par la violance de l'Univers, rendoient quelques sons, qui s'accordoient en une harmonie: comme descrit Alexandre Ephesien.

\section{Ilz rendent touts d'accord une douce harmonie} Quand leurs sons separez d'intervalles certains Ilz resonnent, touchants la lyre en symphonie A sept cordes par ordre en leurs cours plus soudains.

Homere allegoriquement appelle les raions du Soleil fléches, dards ou sagettes. Et n'est céte [141v] invention moins poëtique, c'est à dire, pleine de la fureur des Muses et digne d'admiration, que celle de Platon, lequel attribue à châcun sphere une chantante Sirene. Il me resouvient de l'interpretation que fait Socrate de la chesne d'or du divin Homere, par laquelle il dit qu'il n'entend autre chose, que le Soleil. Car jusques à tant que le circuit et mouvement du Soleil perseverera, toutes les choses tant des Dieux, que des hommes seront et demeureront: mais s'il s'arrestoit comme lié, incontinent tout se dissouldroit, et adviendroit ce qu'on dit vulgairement tout desus desous. ${ }^{53}$ Je recevrai l'opinion de Socrate pour donner autorité à nostre Homere: mais qui entendroit par céte chesne que l'ordre et convenance de l'Univers dépend de la seulle puissance du souverain Dieu, je pense qu'il ne meriteroit moins d'estre oui. Je ne puis passer (bien qu'il me deplaise de vous amuser plus longuement à mes parolles) la description du monde engravée au bouclier ou escu d'Achille, lequel Homere descrit à la figure de l'Univers, laquelle est perfaitement ronde. ${ }^{54}$ Premierement il feint la nuit avoir esté le tems de tout l'ouvrage, representant l'ancienne confusion des choses 
appellée Chaös. Enaprès il fait Vulcan l'ouvrier, lequel (comme Socrate etymologise) signifie le president, ou aiant la charge de la lumiere, ${ }^{55}$ c'est à dire (à mon avis) céte premiere clairté, [142] qui a sorti du Chaös, non encores illustrée des raions du Soleil, laquelle aucuns philosophes escrivent avoir esté mise sous la sphere de la Lune, pour estre le quatriéme et plus subtil element nommé le feu. Et qui voudra tirer de ce discours l'interpretation de la cheute de Vulcan, aura de quoi philosopher. Mais je m'élongne trop du propos, il est tems d'ouir ce que la tailleure de si excellent bouclier decouvroit.

Au bouclier et la terre et la mer et le pol, Le travaillant Soleil et la Lune aussi ronde, Et touts il entailla les Astres, qui au monde Couronnent de splendeurs le ciel de plus hault vol. ${ }^{56}$

Daventage que signifient autre chose les deux villes descrites en ce bouclier, l'une de paix et l'autre de guerre, si non que l'amitié et la dissention sont les commencements des choses, qui a esté l'opinion d'Empedocle aussi poëte Agrigentin? Que reste-il plus? Les cinq bandes ou zones esquelles le ciel est divisé, sont representées en ces vers.

Après que par son art Vulcan a façonné Cinq plis, les deux d'airain, les deux d'estain orné, Mais l'un plus riche d'or. ${ }^{57}$

Ainsi elegamment il appelle plis les zones du monde: par ce qu'on conçoit qu'elles environnent, embrassent et contiennent l'univers à la maniere d'une ceinture. Les deux plus élongnées qui entourent les pols arctique et septentrional et [142v] l'antarctique ou meridional, sont bien comparées à l'airin, duquel la nature est pleine de froid et comme dit en quelque autre lieu le poëte.

Il a le froid airin mordamment empoigné. ${ }^{58}$

Aux deux autres temperées convient la qualité de l'estain, lequel est de matiere humide et entierement traittable. Celle du milieu laquelle le Soleil frappe de ses plus droits raions, et est appellée brulante ou haslée, a beaucoup de resemblance avec l'or. Tout ce que Platon a escrit des enfers et de la condition des morts après céte vie, a esté entierement imité d'Homere. ${ }^{59}$ J'ai le Cratyle en main, lequel seul nous peut rendre certains en quelle opinion Homere a esté envers Platon. Car voulant disputer de la vraie et droite raison des noms sous la personne de Socrate il use de telle entrée, que d'Homere faut apprendre la cause et verité des noms: par ce qu'il distingue les noms donnez et imposez des Dieux, de ceux des hommes: et iceux certainement consistent en nature. ${ }^{60} \mathrm{En}$ lui plus qu'en nul autre apparoissent les traces de toutes les sciences et les exemples des vertus pour bien et heureusement vivre en politique compagnie, ou en l'autorité roiale, ou en l'estat privé. Qui a donc meu Platon de le chasser si rigoreusement? Si nous comparons le dessin de 
l'un avec l'intention de l'autre, il me semble que trouverons quelque moien pour dissoudre aucunement ce doute. Platon se proposant de retirer les mortelz du desordre [143] des pensées et actions humaines, et considerant toutes les citez, les polices, les choses-publiques et les tyrannies tant des Grecs, que des barbares estre entierement corrompuës, il a plus hault elevé son entendement à la congnoissance d'une perfaite republique, de laquelle à son coustume il a cherché l'exemplaire et objet en l'Idée de la Justice divine. Ainsi donc il ne s'est point asservi à descrire les moeurs et manieres de vivre des hommes en ce monde qui tombe sous les sens: ains plustost s'est estudié de les accommoder à la souveraine perfection d'un monde tout intellectuel, selon la conception de son admirable esprit. Il n'a point imité les vulgaires imagiers, lesquelz de toutes parts recueillent les fleurs et traits de beauté, et ingenieusement les enrichissent de diverses couleurs: afin que de plusieurs pourtraits ilz tirent une beauté entiere et accomplie. Mais rejettant toutes les autres polices et choses-publiques il a trouvé au ciel le moule et patron de la sienne, laquelle dès les tendres ongles (comme dient les Grecs) il institue en telle discipline, qu'il ne veut rien apparoir en ses citoiens, qui ne se resente d'une d'une plus haulte et divine pensée. Combien que les poëtes en autres choses soient honnorez et admirez de Platon: toutesfois ilz ne pourroient avoir lieu en sa republique. Car aucuns se rendent trop populaires et semblent estre serfs des afections humaines, les autres et ceux plus graves et excellents (comme Homere) [143v] imitent les moeurs des hommes, qui ont esté ou vertueux ou vicieux, et plus souvent se bigarrent selon les diverses opinions du vulgaire. Daventage Platon, qui n'a rien traitté, que tres-exquisement et en telle majesté que sa grandeur meritoit, s'est tousjours proposé de suivre une vraie et certaine raison, reputant indigne de chatouiller ses citoiens de je nesçai quelles blandices et amorces des fables, desquelles la jeunesse ne peut rapporter aucune utilité. Anacharsis interrogé d'un Athenien si les Scythes avoient l'usage ${ }^{61}$ des fleuttes, respondit, et non aussi des vignes: ${ }^{62}$ par ce que la suite des voluptez est telle, que l'une attire l'autre, lesquelles incontinent épandues font une enveloppe, qui ne peut estre ne deliée, ne rompuë, n'aucunement defaite. En quoi ne se presente nul autre moien de salut, que la fontaine des voluptez seichée, estoupper la source. Ce que Platon a voulu faire en sa republique. Et ne se faut estonner de sa haulte conception. Car pareillement entre ceux, qui ont plus suivi le train des actions humaines esquelles sont infinis erreurs, nous voïons si grande diversité et repugnance de pensées, que les lois des uns sont trop severes et pres-que importables à l'imbecillité des mortelz, les autres trop douces et faciles. Partant comme je ne puis blâmer Platon: ainsi je ne veux rejetter la Muse d'Homere digne d' memoire immortelle, mais non à touts et n'en [144] touts lieux convenable. FAUCHET. Je ne doute 
qu'il ne me seroit plus honnorable de taire en vostre presence, et après si doctes et elegants discours. Mais afin qu'il ne semble qu'en céte compagnie je serve seulement de nombre, je reprendrai le propos que le seigneur Pasquier a traitté le dernier, non que j'aie obstinement juré en l'opinion de Platon: mais je desire sçavoir quelle poësie merite d'estre receue en la chose-publique bien instituée, laquelle en une compagnable tranquillité contente les citoiens. Le premier poinct de céte institution est la discipline et accoustumance de la jeunesse: car en châcune chose le commencement a le premier lieu, et est pres-que le tout: principalement en une molle tendresse, en laquelle est facile d'empraindre telle figure, qu'on veut. Mais (comme dit Pindare) ne le fin regnardeau, ne le terrible Lion pourroit facilement changer la coustume enracinée de nature. ${ }^{63} \mathrm{~A}$ céte cause je nesçai comment céte chose-publique prospereroit, en laquelle les enfants dès le berceau seroient abbreuvez pres-que avec le laict de la nourrice des vaines fables chantées par Hesiode et Homere: comme de celles, qui recitent les guerres et discordes, les adulteres, les risées et moqueries, les surprises, les courroux, les liens et autres semblables mensonges des Dieux. En quelles plus grandes corruptions pourroit [144v] estre enveloppé le jeune esprit, que de l'accoustumer à ouir telles follies? Les uns me diront que la delectation qu'apporte le recit des fables, esveille l'enfant, lequel autrement seroit morne et endormi: les autres que ce qu'Homere et les poëtes ont ainsi chanté, n'est sans grand'allegorie, sous laquelle est couverte une admirable science. Mais ne les uns ne les autres n'ont asses de quoi satisfaire à l'exquise et perfaite institution, laquelle nous desirons. Car ne faut estimer la volupté tant chere, qu'elle soit preferée à la vertu: ains l'esprit bien né dès sa premiere congnoissance se doit tellement façonner à l'amour et plaisir de la vertu, qu'à elle seule, et non à autre il dresse ses pensées. Partant si quelques fables doivent estre racontées à la jeunesse, il faut reciter celles qui sont pleines d'exemples des faits vertueux et memorables. Quant aux autres, on ne les sçauroit tant farder et deguiser d'allegories, qu'elles doivent estre apprises par les enfants, qui ne les peuvent discerner. Je ne m'esbahi donc si Pindare, combien qu'il soit poëte, les blâme et rejette: parce qu'il ne convient dire des Dieux, si non choses honnestes. ${ }^{64}$ Daventage quelle louange merite céte poësie, en laquelle les passions des hommes mal afectionnez sont imitées? Qu'est-il de plus pernicieux exemple, que de representer les sujets dessobeïssants à leur Roi, magistrat, et Capitaine, ou d'exprimer l'avarice, la fureur ou la vo-[145] lupté d'un Prince. Puis qu'Homere se proposoit de donner le premier lieu de son euvre à Achille neveu de Jupiter, devoit-il descrire et mettre devant les yeux d'un peuple Grec les vices de celui, lequel il vouloit faire l'honneur de la Grece? Beaucoup mieux les poëtes Romains ont escrit, lesquelz (comme recite Ciceron en l'oraison pour la loi Manilienne) 
taisoient les vices et calamitez des princes et grands seigneurs: ${ }^{65}$ afin qu'ilz ne feussent congneus du vulgaire, qui en eust fait mal son proufit. Quelle louange peut meriter Homere d'avoir fait son Nestor le plus grand beuveur de l'armée, et chanter de lui à l'heure de l'assault, Nestor a oui le bruit, toutesfois il beuvoit: ${ }^{66}$ Combien qu'il lui attribue la gloire d'estre le plus ancien et meilleur conseillier. Si toutes choses meritoient d'estre honnorées, on pourroit reputer l'imitation d'elles indifferente. Mais la Justice de laquelle depend la societé humaine, ne se peut accompagner d'aucune meschanceté: et partant comme elle excite à imiter la vertu et prudence des anciens, et au contraire à fuïr les vices, qui les font vituperer: ainsi le bon poëte qui rapporte son estude au bien public, ne se doit accommoder à autres descriptions, qu'à celles, qui sont les vraies images de l'honnesteté. Qui fait veoir Achille et Agamemnon s'entr'injuriants, Achille aussi épleuré pour la mort de son compagnon Patrocle, et usant d'extreme cruauté contre les Troiens vaincus: un [145v] Pandare rompant la foi des treves sous l'esperance des dons d'Alexandre filz de Priam, un Ajax desesperé et autres semblables: icelui represente le plus miserable theatre de la vie humaine. Mais si telle est la force de la poësie, que si vivement elle exprime les moeurs et afections des hommes, que nul tant soit-il grave et seure, ne pourroit flechir allieurs sa pensée, qu'en céte part, à laquelle la vehemence poëtique l'auroit émeu et attiré: il me semble que par elle la raison est grandement offensée. Ainsi donc plustost elle donne place aux passions desordonnées, qu'elle les donte et reprime: ce qui est trop élongné de l'excellence des hommes, desquelz qui ne reputeroit indigne de consumer la vie en tristesses, passions et fâcheries pour la perte des choses fortuites et perissables: icelui jetteroit la raison en telz troubles, qu'à grand'peine l'entendement humain se pourroit addresser à la verité. Toutesfois la premiere gloire de la poësie se vante de telz discours: et ainsi par ce qu'elle n'est qu'une imitation, Platon l'appelle troisiéme de la verité, ou plustost le voile, qui masque et deguise icelle de l'abus de touts mensonges. ${ }^{67}$ Car si elle exprime les choses qui sont en nature, elle ne suit que l'art, lequel est seulement l'image de la verité: mais si elle feint et controuve les choses lesquelles ne sont, ou n'est possible d'estre (comme escrit Aristote) ${ }^{68}$ qu'est-ce qu'elle se propose, si non de corrom-[146] pre et obscurcir la verité? Comme le prudent Medecin aiant la congnoissance des herbes et simples medicaments s'arreste plustost à la science fondée sus une certaine raison, qu'à la fortuite experience de je nesçai quelz Empiriques: Ainsi le sage gouverneur de la Chose-publique croira plustost à la doctrine du philosophe qui ne traitte que l'amour de la sagesse, qu'à la volage et inconstante description dispersée par l'euvre du Poëte, lequel ne tend qu'à plaire à la sotte multitude, et partant se change en diverses personnes et contraires afections. Si l'homme a quelque bonté 
de nature et sent un instinct de noblesse, il ne vestira jamais de propos deliberé une personne vile et indigne de soi: ains lui sera fâceux d'en ouïr seulement parler. Mais les Poëtes ne trouvent rien, qui ne soit convenable à leur imitation: et ne donnent moins aux choses vituperables leur grace et bienseance, qu'aux louables et vertueuses. JODELLE. Je n'ai jamais esté si grand admirateur des anciens, que je n'aie quelquefois observé en eux plusieurs fautes, mesmement en la poësie, laquelle je puis dire n'avoir encores esté traitté ne par les Grecs, ne par les Romains, et beaucoup moins par les Italiens et autres estrangers en telle dignité, qu'elle merite. Es uns (je parle des plus excellents) tu trouveras un discours mal commencé, plus [146v] ineptement poursuivi, et sans quelque bonne fin, les causes ou ignorées, ou ignoramment recitées, les comparaisons impropres, messeantes, et trop indignes. Les autres s'éschaufent dès le commencement de je nesçai quelle fureur, mais incontinent leur Muse s'écoulle si basse, qu'elle pert toute gravité. Je reprouve en touts les fables mal inventées, mal disposées et mal accommodées à l'euvre entrepris. Toutesfois j'espere qu'un jour la France verra la poësie au plus hault lustre de sa perfection. PASQUIER. Je ne veux (seigneur Jodelle) entrer en dispute avec toi, pour rendre la memoire des poëtes estrangers plus honnorable. Car la commune amour envers la patrie m'excite à renouveller ton esperance. Aussi (seigneur Fauchet) je t'accorde franchement, qu'es fables anciennes, principalement des Dieux n'est qu'une absurde, inutile et mensongere vanité, indigne d'estre ouie entre les hommes congnoissants un seul et tout-puissant Dieu: duquel les poëtes abusez d'Idolatrie n'ont asses dignement parlé. Mais je ne puis estre tant difficile à l'imitation, que je reprouve celle laquelle artificieusement exprime ce qui est laid et difformé de nature. En quoi le jugement commun fait pour moi. Car non sans grande volupté et admiration nous voions l'image et figure des choses, lesquelles vives et naturelles n'ausons à grand'peine regarder: comme des [147] bestes cruelles et sauvages, des hommes defigurez, et d'autres semblables: non que telles choses apparoissent belles: mais par ce que naivement depeintes et representées de leurs vraies et non feintes couleurs elles semblent avoir quelque vie, et enseignent celui qui s'arreste à elles de leurs formes et figures, lesquelles autrement il ne voudroit ne pourroit congnoistre sans grand desdain et fâcherie. Telle est l'imitation de la poësie, laquelle nous appellons la vive ou parlante peinture, et céte-ci la muëtte poësie. Quand donc les poëtes descrivent quelques choses horribles, quelques faits tristes et miserables, quelques moeurs et afections vehementes, il ne faut tant regarder au sujet, qu'à la bienseance de l'art qui l'a diligemment exprimé. Quelle grace auroit ou la peinture, ou le discours de la chose laide, si elle n'estoit peinte ou descrite selon son naturel? On doit considerer en la Poësie ou les excellentes et admirables sentences desquelles elle 
est tout pleine, ou l'agensement et convenance des personnes et des choses accommodées à l'argument proposé. Quand le poëte descrit le mol et lassif Paris fuiant de la bataille au lict, que nous declaire-il autre chose, que les moeurs d'un homme voluptueux et éfeminé? Toutesfois il ne le propose à imiter, ains plustost à fuir et desestimer. Aussi nous lisons que souvent Homere se mon-[147v]stre offensé des vices, lesquelz l'entreprise de son discours le contraint d'exprimer, et à céte cause les reprend et blâme. Il vitupere l'impudence des parolles d'Agamemnon au prestre, lesquelles semblerent aux Grecs trop furieuses et violantes. Faisant Achille injurier Agamemnon il declaire en ces vers, que rien ne peut estre bien dit ne fait par l'homme irrité.

Encor' le fier Pelide en son audace pire Poursuit Agamemnon et mal donte son ire. ${ }^{69}$

Il est si frequent en Homere, qu'aux recits et descriptions des choses vilaines et deshonnestes il entrelasse son jugement de ce qui se devoit dire ou faire: que je semblerois vouloir imiter un Rhapsode, si je recitois daventage de ses vers. Il convient donc péser la gravité des parolles, lesquelles sont par les poëtes attribuées aux personnes selon la dignité de châcune: et ainsi expliquer leurs sentences qu'elles ne repugnent aucunement à la bienseance et honnesteté. Mais si quelquefois se trouve es mauvais poëtes une absurdité insigne, non pourtant toutesfois la poësie doit estre entierement deprisée. Et n'est le fait de Lycurge digne d'exemple, lequel à cause de l'ivrongnerie de plusieurs arracha toutes les vignes, à l'entour desquelles il se proumenoit: car il lui estoit permis de moderer la fureur du vin par l'attrempance de l'eau. ${ }^{70}$ Puis-que la fragilité humaine est sujette à infinies calamitez et miseres, et n'est la condition de nul tousjours [148] constante et immuable: en quoi sont vituperables les poëtes, s'ilz representent les diverses aventures et contraires desastres? Ilz nous font veoir le cours de nostre vie, n'oubliants rien qui lui puisse estre aucunement proufitable. Si l'homme enclin à quelque vice, le veoit dépeint par le poëte, il rougira incontinent, et en après se contiendra de tomber en semblable malheur, par lequel un autre s'est perdu. A ce propos Timocle comique (comme recite Athenée) asseuroit la tragedie estre pour plusieurs causes utile à la vie humaine: et ainsi a escrit d'elle en la Comedie des celebrants les festes de Denis.

Escoute ami ce que dirai,

$L$ 'homme est sujet de sa nature

A tout travail, et peine dure:

Mais de ce point t'asseurerai,

Qu'il a trouvé à ses ennuits

Pour cher soulas ${ }^{71}$ la tragedie.

Si d'une horreur de maladie

Il est espris, et jours et nuits 
S'afflige, il est lors consolé,

Et peut avoir joie en l'exemple

Des passions, qu'au theatre ample

Il en veoit un plus affollé. ${ }^{72}$

Partant la poësie nous admoneste de deux choses, à sçavoir du vertueux honneur, et de l'infamie deshonneste, icelui pour aimer, céte-ci pour haïr. Aussi elle est utile et à la temperance et [148v] à la haultesse d'esprit. Toutesfois je ne lâche tant la bride à tout ce qu'on peut dire imitation, que je veulle donner trop d'autorité aux vulgaires traductions de je nesçai quelz fabuleux Romans, lesquelz outre les parolles fardées à la volupté des femmes ne contiennent rien que des mensonges: Et serois bien de l'avis d'Alexandre le grand, lequel aiant oui reciter par Aristobule plusieurs menteries feintes en son histoire contre toute semblance de verité jetta le livre au fleuve Hydaspe, et se tournant vers Aristobule lui dit, tu estois aussi digne d'y estre jetté. ${ }^{73}$ FAUCHET. Si l'imitation estoit autre (seigneur Pasquier) que le sujet qui se presente à elle, je n'aurois que dire. Mais penses-tu que nature se laisse vaincre par l'art, et si en elle quelque defaut se trouve, l'imitation le puisse de telles couleurs deguiser, qu'il ne soit tousjours tel, que nature l'a formé. Ciceron dit tres-elegamment que nul art ne peut imiter la subtilité de nature: $:^{74}$ ce qu'avoit escrit Pindare devant lui, comme des-ja il a esté disputé. De quelque fard que tu colores ton opinion, tu ne sçaurois me persuader que celui qui se propose d'exprimer les moeurs et afections voluptueuses et lassives, ne se rende entierement semblable à elles: et pour faire trouver son euvre agreable, ne cherche toutes les manieres, afin d'émouvoir et exciter à semblables cupiditez et passions les hommes, qui perdent quelque peu de leur tems à [149] les ouir, ou lire. Mais (comme dit l'eloquent orateur Demosthene) il ne se peut aucunement faire, que ceux qui s'empeschent aux ${ }^{75}$ choses viles et legeres, soient de noble et hault esprit. ${ }^{76}$ Car quelz sont les dessins et estudes des hommes, telz aussi faut estre leurs esprits. Partant la nature d'un excellent esprit ne peut souffrir, qu'il s'abaisse aux choses moindres et indignes de lui: à quoi toutesfois l'imitation le veut assujettir, si elle a telle autorité que tes fardées et subtiles raisons s'éforcent lui attribuer. RONSARD. Nostre dispute a trouvé plus ample discours, que ne pensois au commencement: telle est la suite des propos, quand unefois ilz tombent es meilleurs esprits, comme sont les vostres. Mais je vous dirai, ce qu'il me semble de toute céte question. Je ne pense aucun douter, que les inventions inspirées de Dieu ne soient incomparablement plus rares, exquises, perfaites et admirables, que celles lesquelles l'imagination humaine feint et conçoit à son plaisir. Mais (comme dit Platon) touts ne sont également favorisez de Dieu. Partant nous pouvons dire que la nature et l'art sont les dons communs de Dieu, sans lesquelz le poëte ne doit esperer de 
rendre quelque euvre louable et excellent. Quant à l'inspiration divine, elle me semble estre un bien plus celeste, duquel châcun ne jouit: ains seulement celui lequel Dieu a voulu plus cherir et decorer: comme [149v] aussi nous disons de la prophetie. Céte poësie donc laquelle est inspirée d'une sainte fureur, ne peut rien imiter qui ne soit tout celeste, illustre et accompli: à l'exemple de laquelle je voudrois que nostre vulgaire ne se proposât que les sujets dignes de quelque honnorable grandeur et vertueuse noblesse. Mais je veoi (messieurs) que nous sommes pres-que demeurez seuls en céte salle, et l'heure presse de nous retirer.

\section{McMaster University}

\section{Notes}

1 Alcibiade II, 147bc (LeC), où Socrate cite un fragment du "Margitès" pseudo-homérique. Le Caron a ajouté une quarantaine de notes marginales que nous avons dû, pour des raisons purement techniques, supprimer dans notre transcription du texte. Nous signalons, cependant, l'existence d'une note marginale de l'auteur à l'aide du sigle: (LeC). Si certaines de ces notes ne font qu'indiquer les arguments abordés par les interlocuteurs (Des fables [132v], De la fureur poëtique [135v], Contre Platon [136v], De la Musique [138], Pourquoi Platon a chassé Homere de sa republicq. [142v], Contre Homere et les semblables poëtes [144], De l'imitation [145v]), la plupart d'entre elles ont pour but de fournir les sources antiques des citations et des idées, tels le nom du dialogue de Platon et le numéro de l'ode de Pindare. Nous avons établi de façon plus précise ces références et nous en avons identifié d'autres.

2 C.-à-d. mais, au contraire.

3 Pausanias, Description de la Grèce I, ii, 3 (LeC). Les allusions homériques se trouvent chez Pausanias. Voir Odyssée VIII, 43 sq. et III, 265 sq.

4 Notre correction d'une faute évidente $-i l$.

5 C.-à-d. entretien, propos.

6 C.à-d. traitez d'une manière douce, tendre.

7 Notre correction d'une faute d'impression - avoi sit.

8 C.-à-d. stylés, habitués.

9 C.à-d. ensuite, par la suite.

10 Voir par exemple Lysis 214a et Banquet 209a.

11 Sur le pouvoir de l'hymne, voir par exemple Olympique VI, 1-4; Néméenne VII, 13-16; Isthmique IV, 38-42.

12 Néméenne VI, 4-7; Olympique VIII, 77-80; Pythique III, 112-115 (LeC).

13 Menon 98c sq. (LeC). Dans le Théagès, indiqué également par l'auteur, il ne s'agit qu'indirectement du rôle de la volonté divine dans l'acquisition de la sagesse (voir 129e).

14 Olympique IX, 28-29; ibid. 100-104; Isthmique III, 4-5; Isthmique V, 11.

15 Parménide 134cd; Cratyle 396ab; Phédon 106d (LeC). Mais voir surtout Lois IV, 716.

16 Isthmique V, 52; Pythique I, 67; Pythique II, 49 et 87-89; Pythique V, 122-123;Pythique X, 10-11; Olympique XIII, 82-83 (LeC).

17 Phédon $78 \mathrm{~b}$ sq.; Timée 28a sq. (LeC).

18 Olympique VII, 24-25; Olympique XII, 7-12; Pythique III, 104-107 (LeC).

19 Phèdre 279c; République VII, 521a. 
20 Pythique II, 56; Pythique VIII, $72-78$ (LeC).

21 Voir Gorgias 523 (LeC), où Platon parle plutôt de la condition des morts que de la mort certaine et inévitable. Pindare est plus explicite. Pythique XII, 29-32; Néméenne VII, 9-20 et 30 (LeC).

22 Olympique II, 34-37.

23 Politique 303ab; République VIII, 555b-562a.

24 Olympique VIII, 23-29.

25 Gorgias 484b (LeC).

26 République I, 331a.

27 Diogène Laërce, Vies des philosophes III, 13-17 (LeC).

28 C.à-d. vantardise.

29 Olympique I, 28-29 (LeC).

30 Xénophon, Mémorables II, i, 21-34 (LeC).

31 Philèbe $16 \mathrm{~cd}(\mathrm{LeC})$.

32 Hymne d Protée (XXV).

33 Cette référence à l'étymologie d'Eidothée se trouve dans Héraclite, Allégories d'Homère 66. Voir Odyssée IV, 365 sq.

34 Cratyle 406a (LeC). L'auteur explique dans la marge: "Platon au Cratyle escrit les Muses estre appellées de $\mu \hat{\omega} \sigma \theta \alpha \iota$, c'est à dire rechercher."

35 Phèdre 245a (LeC).

36 Ion $533 d$.

37 Ion $534 \mathrm{e}$.

38 C.-à-d. excités, émus.

39 Isthmique III, 19 (LeC).

40 République III, 404e et 410a.

41 C.à-d. persuadent.

42 Timée Locrien, De la Nature du monde et de l'âme 82 (LeC).

43 C.-à-d. chanson (avec un senis péjoratif).

44 Deipnosophistes XIV, 631e-632b (LeC).

45 Lachès $188 \mathrm{~d}(\mathrm{LeC})$.

46 Notre correction d'une faute d'impression - trrois.

47 L'auteur explique dans la marge: "De céte question est amplement disputé au premier dialog. du nouv. Pyt." Référence à un autre dialogue de Le Caron qui s'annonce dans la table des matières, "Le nouveau Pytagore, ou des nombres et de l'harmonie," mais dont on ne connaît aucun exemplaire.

48 Cratyle $416 \mathrm{a}-\mathrm{d}$.

49 Ion 530 b.

50 Phédon 94de (LeC). L'allusion homérique se trouve chez Platon. Voir Odyssée XX, 16 sq. L'auteur indique dans la marge les noms d'autres dialogues où Platon cite Homère - Menon, Lois, Théétète.

51 Iliade I, 47 sq. (LeC). Cette citation d'Homère, celle d'Alexandre d'Ephèse, la référence à la Sirène (voir République X, 617b) et la discussion de l'harmonie de l'univers qui s'ensuit se trouvent dans Héraclite, Allégories d'Homère 12.

52 C.-à-d. sans cesse.

53 Thétète $153 \mathrm{~cd}$ (LeC). L'allusion homérique se trouve chez Platon. Voir Miade VIII, $18 \mathrm{sq}$.

54 Iliade XVIII, 477 sq. Cette description allégorique du bouclier d'Achille (avec toutes les citations d'Homère) est tirée presque intégralement d'Héraclite, Allégories d'Homère 43 sq.

55 Cratyle 407c (LeC). Dans le texte de Platon il s'agit d'Héphaistos.

56 lliade XVIII, 483-485. 


\section{8 / Renaissance and Reformation}

57 liade XX, 270-272.

58 niade $\mathrm{V}, 75$.

59 Gorgias 525 de.

60 Cratyle 391 de.

61 Notre correction d'une faute d'impression - usages.

62 Voir Diogène Laërce, Vies des philosophes I, 104.

63 Olympique XI, 19-21 (LeC).

64 Olympique I, 28-36 (LeC).

65 Discours au peuple sur les pouvoirs de Pompée. (Pour la loi Manilia) IX-25.

66 Iliade XIV, 1-2.

67 République X, 599a sq.

68 Art poétique XXIV, 19 [1460a].

69 Miade I, 223-224.

70 Les détails de l'allusion au Lycurgue homérique (voir Iliade VI, 132-137) se trouvent dans Héraclite, Allégories d'Homère 35.

71 C.-à-d. consolation, soulagement.

72 Deipnosophistes VI, 223b-d (LeC).

73 L'anecdote est racontée par Lucien dans "Comment écrire l'histoire" 12.

74 De la Nature des dieux I, xxxiii, 92 (LeC).

75 C.-à-d. s'occupent des.

76 Olynthienne III, 32 (LeC). 